Commun. Korean Math. Soc. 26 (2011), No. 3, pp. 405-416

DOI 10.4134/CKMS.2011.26.3.405

\title{
ON NIL-EXTENSIONS OF LEFT STRONGLY SIMPLE po-SEMIGROUPS
}

\author{
QING SHUN ZHU
}

\begin{abstract}
In this paper, we first introduce the concept of left strongly simple $p o$-semigroups, then we discuss properties and characterizations nil-extensions of left strongly simple $p o$-semigroups and semilattices of left strongly simple $p o$-semigroups. Finally, we give some characterizations of the chain of left strongly simple po-semigroups.
\end{abstract}

\section{Introduction and preliminaries}

The ideal extension problem for semigroups is as follows: Given a semigroup $S$ and a semigroup $Q$ with zero such that $S \cap Q^{*}=\varnothing$ (where $Q^{*}=Q \backslash\{0\}$ ), construct all the semigroups $V$ which have an ideal $K$ which is isomorphic to $S$ and the Rees quotient $V / K$ is isomorphic to $Q$. For ideal extensions of semigroups (without order) have been considered by A. H. Clifford in [4] who gave the first general structure theorem in the case when semigroups is weakly reductive (Theorem 4.21 of [4]). A detailed exposition of the ideal extensions of semigroups can be found in $[5,13]$. Extensions of weakly reductive semigroups, strict and pure extensions, retract extensions, dense extensions, equivalent extensions have been also considered in [13]. Ideal extensions of ordered semigroups have been studied in [10], and nil-extensions of simple ordered semigroups in [3]. We are often interested in building more complex semigroups, lattices, ordered sets, and ordered or topological semigroups out of some of "simple" structure and this can be sometimes achieved by constructing the ideal extensions. The aim of this paper is to study the structure of left strongly simple $p o$-semigroups referring to nil-extension and semilattice of this type of po-semigroups.

Throughout this paper, $Z^{+}$will denote the set of positive integers. As in [1], a po-semigroup (: ordered semigroup) is an ordered set $(S, \leq)$ at the same time a semigroup such that:

$$
a \leq b \Longrightarrow x a \leq x b \text { and } a x \leq b x, \forall a, b, x \in S .
$$

Received March 27, 2010; Revised December 5, 2010.

2010 Mathematics Subject Classification. 20M10, 06F05.

Key words and phrases. left semiregular, left $\pi$-semiregular, left strongly simple, nilextension, semilattices, chain. 
Definition 1.1. Let $S$ be a po-semigroup and $\varnothing \neq A \subseteq S$. $A$ is called a left (right) ideal of $S$ if

(1) $S A \subseteq A(S A \subseteq A)$;

(2) If $a \in A, b \leq a$ with $b \in S$, then $b \in A$.

$A$ is called an ideal of $S$ if $A$ is both a left and right ideal of $S . S$ is left (right) simple if it does not contain proper left (right) ideals. $S$ is simple if $S$ does not contain proper ideals.

Let $S$ be a po-semigroup. For $H \subseteq S$, let $(H]:=\{t \in S \mid t \leq h$ for some $h \in H\}$. For $H=\{a\}$, we write $(a]$ instead of $(\{a\}](a \in S)$. Let $I(a), L(a)$ and $R(a)$ denote the ideal, the left ideal and the right ideal of $S$ generated by an element $a$ of $S$, respectively. One can easily prove that:

$$
\begin{aligned}
L(a) & =(a \bigcup S a], \\
R(a) & =(a \bigcup a S], \\
I(a) & =(a \bigcup S a \bigcup a S \bigcup S a S] .
\end{aligned}
$$

We defined the Green's relation $\mathcal{J}:=\{(x, y) \mid I(x)=I(y)\}$ and $\mathcal{U}_{\mathcal{J}}(S)$ will denote the union of all $\mathcal{J}$-classes of $S$ which are subsemigroups of $S$.

An element $a$ of a po-semigroup $S$ is semisimple if $a \in(S a S a S]$. A set of all semisimple elements of $S$ will be denoted by $\operatorname{Semis}(S)$. A semigroup $S$ is called semisimple if $S=\operatorname{Semis}(S)$.

Lemma 1.1. Let $S$ be a po-semigroup. Then

(1) $S$ is left (right) simple if and only if $S=(S a](S=(a S])$ for every $a \in S$ (see $[6])$.

(2) $S$ is simple if and only if $S=(S a S]$ for every $a \in S$ (see [6]).

(3) $S$ is semisimple if and only if $I(a)=\left(I^{2}(a)\right]$ for every $a \in S$.

Proof. (3) is easy to verify by the definition of semisimple.

Definition $1.2([7,11])$. Let $Y$ be a semilattice. Then $Y$ with the natural order on $Y$ defined by: for $\alpha, \beta \in Y$

$$
\alpha \leq \beta \Leftrightarrow \alpha \beta=\alpha
$$

is a po-semigroup. The po-semigroup $(Y, \cdot, \leq)$ is called a complete semilattice. A po-semigroup $S$ is called a complete semilattice $Y$ of subsemigroups $S_{\alpha}(\alpha \in Y)$ if $S$ is the semilattice $Y$ of subsemigroups $S_{\alpha}(\alpha \in Y)$ such that

$$
(\forall \alpha, \beta \in Y)\left(\forall a \in S_{\alpha}\right)\left(\forall b \in S_{\beta}\right) a \leq b \Rightarrow \alpha \leq \beta .
$$

A congruence determined by the partition $\left\{S_{\alpha} ; \alpha \in Y\right\}$ of $S$ is called a complete semilattice congruence on $S$.

A subsemigroup $F$ of a po-semigroup $S$ is a filter of $S$ if : (1) $a, b \in S, a b \in F$ implies $a \in F$ and $b \in F ;(2) a \in F, b \in S, a \leq b$ implies $b \in F$. The filter of $S$ generated by $x(x \in S)$ is denoted by $N(x)$. Let $\mathcal{N}$ be an equivalence relation defined, as in [6], by 


$$
\mathcal{N}:=\{(x, y) \in S \times S \mid N(x)=N(y)\} .
$$

As in $[7,11]$, for a po-semigroup $S, \mathcal{N}$ is the least complete semilattice congruence on $S$. If $S$ is intra-regular, then we have $\mathcal{N}=\mathcal{J}$ and $S$ is a complete semilattice $Y$ of simple semigroups $S_{\alpha}(\alpha \in Y)$.

As in [2], a relation $\tau$ on a po-semigroup $S$ is defined by:

$$
(a, b) \in \tau \Leftrightarrow\left(\exists x, y \in S^{1}\right) b \leq x a y \text { for every } a, b \in S .
$$

A po-semigroup $S$ is said to have Putcha's property if for $a, b \in S, a \tau b$ implies $a^{2} \tau b^{m}$ for some $m \in Z^{+}$.

Lemma 1.2 (cf. [2, Theorem 2.7]). Let $S$ be a po-semigroup. Then the following conditions are equivalent:

(1) $S$ is a semilattice of Archimedean po-semigroup.

(2) $S$ has Putcha's property.

(3) $(\forall a, b \in S)\left(\exists n \in Z^{+}\right)(a b)^{n} \in\left(S a^{2} S\right]$.

(4) $\mathcal{N}$ is the greatest semilattice congruence on $S$ such that each of its congruence classes is an Archimedean subsemigroup.

If $S$ is a po-semigroup with zero 0 , we will write $S=S^{0}$. An element $a$ of $S$ is called nilpotent if there exists $n \in Z^{+}$such that $a^{n}=0$. The set of all nilpotents of $S$ is denoted by $\operatorname{Nil}(S)$. A semigroup $S$ is a nil-semigroup if $S=\operatorname{Nil}(S)$. Let $K$ be a proper ideal of a po-semigroup $S$. Then

$$
\rho_{K}=(K \times K) \bigcup 1_{S}
$$

is a semigroup congruence on $S$. The quotient semigroup

$$
S / \rho_{K}=\{K\} \bigcup\{\{x\} \mid x \in S \backslash K\}
$$

is the Rees factor semigroup of $S$ modulo $K$ and is normally written by $S / K$ rather than $S / \rho_{K}$. We may describe $S / K$ as the result of collapsing $K$ into a single (zero) element, while the elements of $S$ outside of $K$ retain their identity. A simple argument shows that $S / K$ with an order " $\leq_{K}$ " defined by: for $a=$ $\{x\}, b=\{y\}, x, y \in S \backslash K$,

$$
a \leq_{K} b \Leftrightarrow x \leq_{K} y, K \leq_{K} a \Leftrightarrow(\exists z \in K) z \leq x
$$

is a po-semigroup with zero $K$.

Definition 1.3. Let $K$ be an ideal of a po-semigroup $S$. Then $\left(S / K, \cdot, \leq_{K}\right)$ is called the Rees factor semigroup of $S$ modulo $K$, and $S$ is called an ideal extension of $K$ by the po-semigroup $S / K$. An ideal extension $S$ of $K$ is called a nil-extension of $K$ if $S / K$ is a nil po-semigroup.

By Definition 1.3, we can easily prove the following lemma (see [3]).

Lemma 1.3. Let $S$ be a po-semigroup and $K$ be an ideal of $S$. Then the following conditions are equivalent:

(1) $S$ is a nil-extension of $K$.

(2) $(\forall a \in S)\left(\exists m \in Z^{+}\right) a^{m} \in K$.

Example 1.1. We consider the set $S=\{a, b, c, d, f\}$, defined by multiplication and the order below [8]: 


\begin{tabular}{c|c|c|c|c|c}
$\cdot$ & $a$ & $b$ & $c$ & $d$ & $f$ \\
\hline$a$ & $a$ & $b$ & $c$ & $d$ & $c$ \\
\hline$b$ & $a$ & $b$ & $c$ & $d$ & $a$ \\
\hline$c$ & $a$ & $b$ & $c$ & $d$ & $c$ \\
\hline$d$ & $d$ & $d$ & $d$ & $d$ & $d$ \\
\hline$f$ & $a$ & $b$ & $c$ & $d$ & $c$
\end{tabular}

$\leq:=\{(a, a),(a, d),(b, b),(b, d),(c, a),(c, c),(c, d),(c, f),(d, d),(f, f)\}$.

For an easy way to check that $S$ is a po-semigroup, we refer to [8]. We give the covering relation and the figure of $S$ :

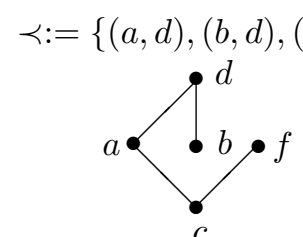

The ideals of $S$ are the sets: $\{a, b, c, d\}$ and $S$. Let $K=\{a, b, c, d\}$. Since $f^{2}=c \in K$, by Lemma 1.3, it is obvious that $S$ is a nil-extension of $K$.

Definition 1.4 ([14, 12]). An element $a$ of a po-semigroup $S$ is a left (right) semiregular element if $a \leq x a y a\left(a \leq a x^{\prime} a y^{\prime}\right)$ for some $x, y, x^{\prime}, y^{\prime} \in S$.

Equivalent Definition: $a \in(S a S a](a \in(a S a S])$ for every $a \in S$ [14].

The set of all left (right) semiregular elements of $S$ will be denoted by $L S R e g(S)(R S \operatorname{Reg}(S)) . S$ is called left (resp. right) semiregular if $S=L S \operatorname{Reg}(S)$ $(S=R S \operatorname{Reg}(S)) . S$ is called semiregular if $S=L S \operatorname{Reg}(S) \cap R S \operatorname{Reg}(S)$.

Lemma 1.4 ([14]). Let $S$ be a po-semigroup. Then the following conditions are equivalent:

(1) $S$ is left semiregular.

(2) $\left(L^{2}\right]=L$ for every left ideal $L$ of $S$.

(3) $L(a) \bigcap L(b) \subseteq(L(a) L(b)]$ for every $a, b \in S$.

Definition 1.5. Let $S$ be a po-semigroup, $S$ is called left (right) strongly simple if $S$ is simple and left (right) semiregular. $S$ is called strongly simple if $S$ is both left and right strongly simple.

Example 1.2. We consider the ordered semigroup $S=\{a, b, c, d, e\}$, defined by multiplication and the order below [9]:

\begin{tabular}{c|c|c|c|c|c}
$\cdot$ & $a$ & $b$ & $c$ & $d$ & $e$ \\
\hline$a$ & $a$ & $a$ & $c$ & $a$ & $c$ \\
\hline$b$ & $a$ & $a$ & $c$ & $a$ & $c$ \\
\hline$c$ & $a$ & $a$ & $c$ & $a$ & $c$ \\
\hline$d$ & $d$ & $d$ & $e$ & $d$ & $e$ \\
\hline$e$ & $d$ & $d$ & $e$ & $d$ & $e$
\end{tabular}




$$
\begin{aligned}
\leq:=\{ & (a, a),(a, b),(a, c),(a, d),(a, e),(b, b),(b, c),(b, d), \\
& (b, e),(c, c),(c, e),(d, d),(d, e),(e, e)\} .
\end{aligned}
$$

We give the covering relation and the figure of $S$ :

$$
\prec:=\{(a, b),(b, c),(b, d),(c, e),(d, e)\},
$$

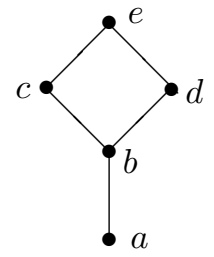

For any $x \in S$, it is enough to prove that $(S x S]=S$. Hence, $S$ is simple. For an easy way to check that $S$ is left semiregular and right semiregular. Thus $S$ is both left and right strongly simple. Hence $S$ is a strongly simple $p o$-semigroup.

Now, we generalize the concepts of (intra-, left, right) $\pi$-regular semigroups without order to $p o$-semigroups. Let $S$ be a po-semigroup. The set of all regular (left regular, right regular, intra-regular) elements of $S$ is denoted by $\operatorname{Reg}(S)(\operatorname{LReg}(S), \operatorname{RReg}(S), \operatorname{Intra}(S))$.

Definition 1.6. A po-semigroup $S$ is called $\pi$-regular (left $\pi$-regular, right $\pi$-regular, intra- $\pi$-regular, left $\pi$-semiregular) if for every $a \in T$ there exists $n \in Z^{+}$such that $a^{n} \in \operatorname{Reg}(S)(\operatorname{LReg}(S), \operatorname{RReg}(S), \operatorname{Intra}(S), \operatorname{LSReg}(S))$.

Definition 1.7. A po-semigroup $S$ is called Archimedean, if for any $a, b \in S$ there exists $n \in Z^{+}$such that $a^{n} \in(S b S]$.

\section{Nil-extensions of left strongly simple po-semigroups}

Now we are ready to prove the main results of this paper. First, we introduce a lemma which will be used frequently in this paper.

Lemma 2.1. Let po-semigroup $S$ be a complete semilattice $Y$ of subsemigroups $S_{\alpha}(\alpha \in Y)$. Then

(1) the following conditions hold:

$$
\begin{gathered}
\operatorname{LSReg}(S)=\bigcup_{\alpha \in Y} \operatorname{LSReg}\left(S_{\alpha}\right), \operatorname{Semis}(S)=\bigcup_{\alpha \in Y} \operatorname{Semis}\left(S_{\alpha}\right), \\
\operatorname{Intra}(S)=\bigcup_{\alpha \in Y} \operatorname{Intra}\left(S_{\alpha}\right), \operatorname{LReg}(S)=\bigcup_{\alpha \in Y} \operatorname{LReg}\left(S_{\alpha}\right) .
\end{gathered}
$$

(2) $S$ is left semiregular (semisimple, intra-regular, left regular) if and only if $S_{\alpha}$ is left semiregular (semisimple, intra-regular, left regular) for all $\alpha \in Y$.

(3) $S$ is left $\pi$-semiregular ( $\pi$-semisimple, intra $\pi$-regular, left $\pi$-regular) if and only if $S_{\alpha}$ is left $\pi$-semiregular ( $\pi$-semisimple, intra $\pi$-regular, left $\pi$ regular) for all $\alpha \in Y$. 
Proof. (1) It is obvious that $\operatorname{LSReg}(S) \supseteq \bigcup_{\alpha \in Y} \operatorname{LSReg}\left(S_{\alpha}\right)$. Let $a \in \operatorname{LSReg}(S)$. Then there exists $\alpha \in Y$ and $u \in S_{\beta}, v \in S_{\gamma}$ such that $a \in S_{\alpha}$ and $a \leq$ uava. Since $Y$ is a complete semilattice of $S$, we have $\alpha \leq \beta \alpha \gamma \alpha$. From $\beta \alpha \gamma \alpha \leq \beta \alpha \gamma \leq \beta \alpha \leq \alpha$, which implies $\alpha=\alpha \beta \gamma$. Since $a \leq$ uava $\leq$ $u(u a v a) v(u a v a)=\left(u^{2} a v\right) a(v u a v) a$, in which $u^{2} a v, v u a v \in S_{\beta \alpha \gamma}=S_{\alpha}$, we have that $a \in \operatorname{LSReg}\left(S_{\alpha}\right)$. Thus it implies that $\operatorname{LSReg}\left(S_{\alpha}\right) \subseteq \bigcup_{\alpha \in Y} \operatorname{LSReg}\left(S_{\alpha}\right)$. Hence, the first equality in (1) holds. Similar arguments can show that the other equalities in (1) hold.

Both (2) and (3) are immediate consequences of (1).

Theorem 2.1. The following conditions on a po-semigroup $S$ are equivalent:

(1) $S$ is left strongly simple.

(2) $(\forall a, b \in S) a \in(S b S a]$.

(3) Every left ideal of $S$ is a simple po-semigroup.

(4) $S$ is simple and $\left(L^{2}\right]=L$ for every left ideal $L$ of $S$.

(5) $S$ is simple and every left ideal of $S$ is an intra-regular po-semigroup.

Proof. $(1) \Rightarrow(2)$ and $(2) \Rightarrow(3)$ It follows immediately from Lemma 1.1 and the definition of left strongly simple.

$(3) \Rightarrow(4)$ Since $S$ is a left ideal of $S$ itself. By hypothesis, $S$ is simple. Let $L$ be a left ideal of $S$. It is obvious that $\left(L^{2}\right] \subseteq L$. Let $a \in L$. Then $L(a)=(L(a) a L(a)] \subseteq\left(L^{2}(a)\right] \subseteq\left(L^{2}\right]$. It implies that $L \subseteq\left(L^{2}\right]$. Therefore, we have $\left(L^{2}\right]=L$.

$(4) \Rightarrow(5)$ Let $L$ be a left ideal of $S$ and $a \in L$. By hypothesis, we have $a^{3} \in L$ and $S=\left(S a^{3} S\right]$. Thus

$$
a \in L=\left(L^{2}\right] \subseteq(S L] \subseteq\left(\left(S a^{3} S\right] L\right] \subseteq\left(S L a^{2} L\right] \subseteq\left(L a^{2} L\right]
$$

which shows that $L$ is an intra-regular po-semigroup.

$(5) \Rightarrow(1)$ Let $a \in S$, by hypothesis, we have $a \in L(a)=\left(L(a) a^{2} L(a)\right] \subseteq$ $\left((a \bigcup S a] a^{2}(a \bigcup S a]\right] \subseteq(S a S a]$. Thus, $S$ is left semiregular.

Theorem 2.2. The following conditions on a po-semigroup $S$ are equivalent:

(1) $S$ is a semilattice of left strongly simple po-semigroup.

(2) $(\forall a \in S) a \in\left(S a^{n} S a\right]$ for every $n \in Z^{+}$.

(3) $(\forall a \in S) a \in\left(S a^{2} S a\right]$.

(4) Every left ideal of $S$ is an intra-regular subsemigroup of $S$.

(5) Every left ideal of $S$ is a semisimple subsemigroup of $S$.

Proof. (1) $\Rightarrow(2)$ Suppose $S$ is a semilattice of $Y$ subsemigroups $S_{\alpha}(\alpha \in Y)$ which are left strongly simple. Let $a \in S_{\alpha}$ for some $\alpha \in Y$. Then $a^{n} \in S_{\alpha}$ for every $n \in Z^{+}$. Since $S_{\alpha}$ is left strongly simple, by Theorem 2.1, we have $a \in\left(S_{\alpha} a^{n} S_{\alpha} a\right] \subseteq\left(S a^{n} S a\right]$.

$(2) \Rightarrow(3)$ It is obvious.

$(3) \Rightarrow(4)$ Let $L$ be a left ideal of $S$ and $a \in L$. By hypothesis, we have

$$
a \in\left(S a^{2} S a\right] \subseteq\left(S\left(S a^{4} S a^{2}\right] S a\right] \subseteq\left(S a^{4} S a^{2} S a\right] \subseteq\left(S L a^{2} S L\right] \subseteq\left(L a^{2} L\right] .
$$


Thus $L$ is an intra-regular subsemigroup of $S$.

$(4) \Rightarrow(5)$ In view of the hypothesis, a simple argument shows that this is evident.

$(5) \Rightarrow(1)$ Let $a \in S$. In view of the hypothesis, we have $a \in(L(a) a L(a) a L(a)]$ $\subseteq\left(S a^{2} S a\right]$. Thus $S$ is an intra-regular and left semiregular. It implies $S$ is a complete semilattice $Y$ of simple semigroup $S_{\alpha}(\alpha \in Y)$. By Lemma 2.1, $S_{\alpha}$ is left semiregular, so $S_{\alpha}$ is left strongly simple.

The intersection of all ideals of a po-semigroup $S$, if nonempty, is called the kernel of $S$ and denoted by $K(S)$. A po-semigroup $S$ is called completely regular if $S$ is regular, left regular, right regular po-semigroup [15]. In [15], we have shown that $S$ is completely regular if and only if $\forall a \in S, a \in\left(a^{2} S a^{2}\right]$, equivalently, $S$ is a union of disjoint $B$-simple subsemigroups of $S$.

Lemma $2.2([3])$. Let $S$ be an Archimedean po-semigroup. If $\operatorname{Intra}(S) \neq \varnothing$, then

(1) $S$ has a kernel $K(S)$ such that

$$
\forall a \in \operatorname{Intra}(S), K(S)=(S a S], \operatorname{Intra}(S) \subseteq K(S) .
$$

(2) $S$ is a nil-extension of simple po-semigroup $K(S)$.

Lemma 2.3 ([16]). Let $S$ be a po-semigroup. Then kernel $K(S)$ of $S$ is a completely regular simple subsemigroup.

Theorem 2.3. The following conditions on a po-semigroup $S$ are equivalent:

(1) $S$ is a nil-extension of a left strongly simple po-semigroup.

(2) $(\forall a, b \in S)\left(\exists n \in Z^{+}\right) a^{n} \in\left(S b^{m} S a^{n}\right]$ for every $m \in Z^{+}$.

(3) $(\forall a, b \in S)\left(\exists n \in Z^{+}\right) a^{n} \in\left(S b S a^{n}\right]$.

(4) $S$ is Archimedean and left $\pi$-semiregular.

Proof. (1) $\Rightarrow(2)$ Let $S$ be a nil-extension of a left strongly simple po-semigroup $K$. Let $a, b \in S$. Then $a^{n} \in K$ for some $n \in Z^{+}$. Since $K$ is an ideal of $S$, we have $a^{n} b^{m} \in K S \subseteq K$ for every $m \in Z^{+}$. But $K$ is left strongly simple, by Theorem2.1, for $a^{n}, a^{n} b^{m}$, we have $a^{n} \in\left(S a^{n} b^{m} S a^{n}\right] \subseteq\left(S b^{m} S a^{n}\right]$.

$(2) \Rightarrow(3) \Rightarrow(4)$ The implcations follow immediately.

(4) $\Rightarrow(1)$ Suppose $S$ is Archimedean and left $\pi$-semiregular. Let $a \in S$. Then there exist $x, y \in S$ and $n \in Z^{+}$such that $a^{n} \leq x a^{n} y a^{n} \leq\left(x a^{n} y\right)^{m} a^{n}$ for every $m \in Z^{+}$. Since $S$ is Archimedean, for $x a^{n} y$ and $a^{2 n}$ there exist $k \in Z^{+}$ and $u, v \in S$ such that $\left(x a^{n} y\right)^{k} \leq u a^{2 n} v$. Now

$$
a^{n} \leq x a^{n} y a^{n} \leq\left(x a^{n} y\right)^{k} a^{n} \leq u a^{2 n} v a^{n}=u a^{2 n}\left(v a^{n}\right) \Rightarrow a^{n} \in\left(S a^{2 n} S\right]
$$

which shows that $a^{n} \in \operatorname{Intra}(S)$. Hence $\operatorname{Intra}(S) \neq \varnothing$. By Lemma 2.2, we conclude that $S$ is a nil-extension of simple po-semigroup $K(S)$. By Lemma 2.3, $K(S)$ is a completely regular simple subsemigroup. A simple argument shows that $K(S)$ is left semiregular. Hence $S$ is a nil-extension of a left strongly simple po-semigroup. 
Lemma 2.4 ([3]). Let $S$ be a po-semigroup. Then $S$ is Archimedean and intra-regular if and only if $S$ is simple.

Theorem 2.4. The following conditions on a po-semigroup $S$ are equivalent:

(1) $S$ is a semilattice of nil-extensions of left strongly simple po-semigroups.

(2) $(\forall a, b \in S)\left(\exists n \in Z^{+}\right)(a b)^{n} \in\left(S a^{m} S(a b)^{n}\right]$ for every $m \in Z^{+}$.

(3) $(\forall a, b \in S)\left(\exists n \in Z^{+}\right)(a b)^{n} \in\left(S a^{2} S(a b)^{n}\right]$.

(4) $S$ is intra $\pi$-regular and $\operatorname{LSReg}(S)=\operatorname{Intra}(S)=\operatorname{Semis}(S)=\mathcal{U}_{\mathcal{J}}(S)$.

Proof. (1) $\Rightarrow(2)$ Suppose $S$ is a semilattice $Y$ of subsemigroups $S_{\alpha}(\alpha \in Y)$ which are nil-extensions of left strongly simple po-semigroup. Let $a \in S_{\alpha}, b \in$ $S_{\beta}$ for some $\alpha, \beta \in Y$. Then $a b \in S_{\alpha \beta}, a^{m} b \in S_{\alpha \beta}$ for every $m \in Z^{+}$, so that there exists $n \in Z^{+}$such that

$$
(a b)^{n} \in\left(S_{\alpha \beta} a^{m} b S_{\alpha \beta}(a b)^{n}\right] \subseteq\left(S a^{m} S(a b)^{n}\right]
$$

by Theorem 2.3 .

$(2) \Rightarrow(3)$ It is obvious.

$(3) \Rightarrow(4)$ Let $a \in S$. By hypothesis, there exist $x, y \in S$ and $n \in Z^{+}$such that $a^{2 n} \leq x a^{2} y a^{2 n} \leq x\left(a^{2} y x\right)^{m} a^{2} y a^{2 n}$ for every $m \in Z^{+}$. But for $a^{2}, y x$ there exist $u_{1}, v_{1} \in S$ and $k_{1} \in Z^{+}$such that $\left(a^{2} y x\right)^{k_{1}} \leq u_{1} a^{4} v_{1}\left(a^{2} y x\right)^{k_{1}}$. So we have

$$
a^{2 n} \leq x\left(a^{2} y x\right)^{k_{1}} a^{2} y a^{2 n} \leq x u_{1} a^{4} v_{1}\left(a^{2} y x\right)^{k_{1}} a^{2} y a^{2 n} \in\left(S a^{4} S a^{2 n}\right] .
$$

Hence there exist $u_{2}, v_{2} \in S$ such that $a^{2 n} \leq u_{2} a^{4} v_{2} a^{2 n} \leq u_{2}\left(a^{4} v_{2} u_{2}\right)^{k_{2}} a^{4} v_{2} a^{2 n}$ for every $k_{2} \in Z^{+}$. Continuing this way, then there exist $u_{2 n}, v_{2 n} \in S$ such that $a^{2 n} \leq u_{2 n} a^{4 n} v_{2 n} a^{2 n} \in\left(S a^{4 n} S a^{2 n}\right]$, which shows that $S$ is intra $\pi$-regular and left $\pi$-semiregular.

Let $a \in \operatorname{Semis}(S)$. Then $(a)_{\mathcal{J}}$ is a subsemigroup of $S$. In fact: Let $b, c \in$ $(a)_{\mathcal{J}}$, then $I(a)=I(b)=I(c)$. A simple argument shows that $I(b c) \subseteq I(a)$. Conversely, since $a \in \operatorname{Semis}(S)$, then $a \in($ SaSaS $] \subseteq($ SaSaSaSaSaS $] \subseteq$ $\left(I^{5}(b)\right] \subseteq(S b S]$. Similarly, $a \in(S c S)$. Thus we have $a \in(S a S a S] \subseteq$ $(S(S c S] S(S b S] S] \subseteq(S c S b S]$. Then there exist $x, y, z \in S$ such that $a \leq x c y b z$. Since $a \in \operatorname{Semis}(S)$, there exist $u, v, d \in S$ such that for every $n \in Z^{+}$

$$
\begin{aligned}
a & \leq \text { uavad } \leq u^{n+1} a(\text { vad })^{n+1} \leq u^{n+1} a(\text { vxcybzd })^{n+1} \\
& =u^{n+1} a v x(\operatorname{cyb} z d v x)^{n} c y b z d .
\end{aligned}
$$

But for $c y b, z d v x$, by hypothesis, there exist $p, q \in S$ and $m \in Z^{+}$such that

$$
\begin{aligned}
(c y b z d v x)^{m} & \leq p(c y b)^{2} q(c y b z d v x)^{m}=(p c y) b c(y b q)(c y b z d v x)^{m} \\
& \in S I(b c) S \subseteq I(b c) .
\end{aligned}
$$

Hence $I(a) \subseteq I(b c)$, so $I(a)=I(b c)$, it implies $b c \in(a)_{\mathcal{J}}$. Thus $(a)_{\mathcal{J}}$ is a subsemigroup of $S$ and $\operatorname{Semis}(S) \subseteq \mathcal{U}_{\mathcal{J}}(S)$ is obvious by $a \in(a)_{\mathcal{J}} \in \mathcal{U}_{\mathcal{J}}(S)$.

On the other hand, let $a \in \mathcal{U}_{\mathcal{J}}(S)$, then there exists $b \in S$ such that $a \in$ $(b)_{\mathcal{J}} \in \mathcal{U}_{\mathcal{J}}(S)$. Since $(b)_{\mathcal{J}}$ is a subsemigroup of $S$, then $a^{4} \in(b)_{\mathcal{J}}$. Hence $I\left(a^{4}\right)=I(b)=I(a)$, so we have $a \in I\left(a^{4}\right) \subseteq\left(S a^{2} S\right] \subseteq(S a S a S]$, i.e., $a \in$ 
$\operatorname{Semis}(S)$ and $a \in \operatorname{Intra}(S)$. Thus $\mathcal{U}_{\mathcal{J}}(S) \subseteq \operatorname{Semis}(S)$ and $\mathcal{U}_{\mathcal{J}}(S) \subseteq \operatorname{Intra}(S)$, so that $\operatorname{Semis}(S)=\mathcal{U}_{\mathcal{J}}(S)$. By $\operatorname{Semis}(S)=\mathcal{U}_{\mathcal{J}}(S)$, a simple argument shows that $L S \operatorname{Reg}(S) \subseteq \operatorname{Semis}(S)=\mathcal{U}_{\mathcal{J}}(S)$ and $\operatorname{Intra}(S) \subseteq \operatorname{Semis}(S)=\mathcal{U}_{\mathcal{J}}(S)$. Thus $\operatorname{Intra}(S)=\mathcal{U}_{\mathcal{J}}(S)$.

By hypothesis, it is obvious that $S$ has Putcha's property. In view of Lemma $1.2, \mathcal{N}$ is the greatest semilattice congruence on $S$ such that $(x)_{\mathcal{N}}$ is an Archimedean subsemigroup for every $x \in S$. But $\mathcal{N}$ is the least complete semilattice congruence on $S$. Since $S$ is left $\pi$-semiregular and intra $\pi$-regular, by Lemma $2.1,(x)_{\mathcal{N}}$ is left $\pi$-semiregular and intra $\pi$-regular. From this it follows by Theorem 2.3 that $(x)_{\mathcal{N}}$ is a nil-extensions of left strongly simple po-semigroup $K_{x}$ for every $x \in S$. Let $c \in \operatorname{Intra}(S)$. Then $c \in$ $\operatorname{Intra}\left((c)_{\mathcal{N}}\right)$, i.e., there exist $u, v \in(c)_{\mathcal{N}}$ such that $c \leq u c^{2} v \leq u^{k} c(c v)^{k}$ for every $k \in Z^{+}$. For $u, c v$ there exists $n \in Z^{+}$such that $u^{n},(c v)^{n} \in K_{c}$. Hence $c \leq u^{n} c(c v)^{n} \in K_{c} c K_{c} \subseteq K_{c} \subseteq \operatorname{LSReg}(S)$. Thus, Intra $(S) \subseteq \operatorname{LSReg}(S)$. We have $\operatorname{Intra}(S)=\operatorname{LSReg}(S)$.

(4) $\Rightarrow(1)$ Let $a, b \in S$. By $S$ is intra $\pi$-regular, then there exist $u, v \in S$ and $n \in Z^{+}$such that $(a b)^{n} \leq u(a b)^{2 n} v \in\left(S(b a)^{n+1} S\right]$ and $(b a)^{n+1} \in\left(S(a b)^{n} S\right]$, which implies that $(a b)^{n},(b a)^{n+1} \in\left((a b)^{n}\right)_{\mathcal{J}}$. Since $(a b)^{n} \in \operatorname{Intra}(S)=$ $\operatorname{Semis}(S)$, the process is similar to the proof of $(3) \Rightarrow(4)$, we have $\left((a b)^{n}\right)_{\mathcal{J}}$ is a subsemigroup of $S$. Then $(b a)^{n+1}(a b)^{n} \in\left((a b)^{n}\right)_{\mathcal{J}}$. Now

$$
(a b)^{n} \in I\left((b a)^{n+1}(a b)^{n}\right)=I\left((b a)^{n} b a^{2} b(a b)^{n-1}\right) \subseteq\left(S a^{2} S\right] .
$$

Thus $S$ has Putcha's property by Lemma 1.2. By Lemma 1.2, $\mathcal{N}$ is the greatest semilattice congruence on $S$ and the $\mathcal{N}$-class $(a)_{\mathcal{N}}$ of $S$ containing $a$ is an Archimedean subsemigroup for every $a \in S$. Since $\mathcal{N}$ is the least complete semilattice congruence on $S$, and $S$ is intra $\pi$-regular, by Lemma $2.1,(a)_{\mathcal{N}}$ is intra $\pi$-regular. Let $b \in(a)_{\mathcal{N}}$, then there exists $m \in Z^{+}$such that $b^{m} \in \operatorname{Intra}\left((a)_{\mathcal{N}}\right) \subseteq \operatorname{Intra}(S)=\operatorname{LSReg}(S)$. By Lemma 2.1, then $b^{m} \in \operatorname{LSReg}\left(\left(b^{m}\right)_{\mathcal{N}}\right)$. Since $(a)_{\mathcal{N}}$ is a subsemigroup, we have $b^{m} \in(a)_{\mathcal{N}}$, thus $\left(b^{m}\right)_{\mathcal{N}}=(a)_{\mathcal{N}}$, so we get $(a)_{\mathcal{N}}$ is a left $\pi$-semiregular po-semigroup. Since $(a)_{\mathcal{N}}$ is Archimedean left $\pi$-semiregular po-semigroup. By Theorem 2.3, $(a)_{\mathcal{N}}$ is nil-extensions of left strongly simple po-semigroups.

\section{Chain of left strongly simple po-semigroups}

Further, we will consider chains of left strongly simple po-semigroups.

For a po-semigroup $S, \sigma$ a semilattice congruence on $S$, we denote by " $\preceq$ " the order on the semigroup $S / \sigma=\left\{(x)_{\sigma} \mid x \in S\right\}$ defined by:

$$
(x)_{\sigma} \preceq(y)_{\sigma} \Leftrightarrow(x)_{\sigma}=(x y)_{\sigma}
$$

$(S / \sigma, \cdot, \preceq)$ is a po-semigroup.

Definition 3.1. Let $S$ be a po-semigroup. $S$ is called a chain of left strongly simple po-semigroups if there exists a semilattice congruence $\sigma$ on $S$ such that $(x)_{\sigma}$ is a left strongly simple po-subsemigroup of $S$ for every $x \in S$ and $(S / \sigma, \preceq)$ is a chain. 
Theorem 3.1. Let $S$ be a po-semigroup, $\mathcal{C}(S)$ the set of ideals of $S$. The following conditions are equivalent:

(1) $S$ is a chain of left strongly simple semigroups.

(2) $(\forall a, b \in S) a \in(S a b S a]$ or $b \in(S a b S b]$.

(3) $(\forall a \in S) a \in\left(S a^{2} S a\right]$ and every ideal of $S$ is prime.

(4) $(\forall a \in S) a \in\left(S a^{2} S a\right]$ and $(\mathcal{C}(S), \subseteq)$ is a chain.

Proof. (1) $\Rightarrow(2)$ Let $\sigma$ be a semilattice congruence of $S$ such that $(x)_{\sigma}$ is a left strongly simple semigroup of $S$ for every $x \in S$ and $(S / \sigma, \preceq)$ is a chain. Let $a, b \in S$. For $(a)_{\sigma},(b)_{\sigma}$, we have $(a)_{\sigma} \preceq(b)_{\sigma}$ or $(b)_{\sigma} \preceq(a)_{\sigma}$. If $(a)_{\sigma} \preceq(b)_{\sigma}$, then $a, a b \in(a)_{\sigma}$, and by Theorem 2.1 , we have $a \in\left((\bar{a})_{\sigma} a b(a)_{\sigma} a\right] \subseteq(\overline{S a b S a}]$. If $(b)_{\sigma} \preceq(a)_{\sigma}$, similar arguments can show that $b \in(S a b S b]$.

$(2) \Rightarrow(3)$ It is obvious.

$(3) \Rightarrow(4)$ Let $\mathcal{C}(S)$ be the set of ideals of $S$. Let $A, B \in \mathcal{C}(S)$ such that $A \nsubseteq B$. We prove that $B \subseteq A$. Let $a \in A, a \notin B$ and $b \in B$. Then $a b \in A B \subseteq A, B$. In view of hypothesis, we have $a b \in\left(S(a b)^{2} S a b\right] \subseteq(S a b S]$. Since $(S a b S]$ is an ideal of $S$, we have $a \in(S a b S]$ or $b \in(S a b S]$. If $a \in(S a b S]$, then $a \in(S a b S] \subseteq(S B S] \subseteq(B]=B$, impossible. Thus $b \in(S a b S] \subseteq(S A S] \subseteq$ $(A]=A$.

$(4) \Rightarrow(1)$ In view of the hypothesis, it is clear that $S$ is intra-regular. By [13, Theorem 2], there exists a complete semilattice congruence $\sigma$ of $S$ such that $(x)_{\sigma}$ is a simple po-semigroup of $S$ for every $x \in S$ and $(S / \sigma, \preceq)$ is a chain. Let $a \in(x)_{\sigma}$. By hypothesis, we have $a \in\left(S a^{2} S a\right]$. Assume that $u, v \in S$ such that $a \leq u a^{2} v a$. Then, taking into account the fact that $\sigma$ is complete, we have $\left(a, a u a^{2} v a\right) \in \sigma$. Since $\sigma$ is a semilattice congruence on $S$, we have $\left(a u a^{2} v a, a^{2} u a^{2} v\right) \in \sigma$. Since $\left(a^{2}, a\right) \in \sigma$, we have $\left(a u a^{2} v a, a u a^{2} v\right) \in \sigma$. Then, since $\left(u, u^{2}\right) \in \sigma$, we have $\left(a u a^{2} v, a u^{2} a^{2} v\right) \in \sigma$. Then, since $\left(a u^{2} a^{2} v, u a u a^{2} v\right) \in$ $\sigma$, we have $\left(a u a^{2} v a, u a u a^{2} v\right) \in \sigma$. Therefore, we get that $\left(a, u a u a^{2} v\right) \in \sigma$ and $u a u a^{2} v \in(a)_{\sigma}$. Analogously, $v a u a^{2} v \in(a)_{\sigma}$. Now

$$
a \leq u a^{2} v a \leq u a\left(u a^{2} v a\right) v a \leq\left(u a u a^{2} v\right) a v a\left(u a^{2} v a\right)=\left(u a u a^{2} v\right) a\left(v a u a^{2} v\right) a
$$

in which $u a u a^{2} v, v a u a^{2} v \in(a)_{\sigma}$, we shows that $(x)_{\sigma}$ is a left semiregular subsemigroup. Thus, $(x)_{\sigma}$ is a left strongly simple subsemigroup.

Theorem 3.2. The following conditions on a po-semigroup $S$ are equivalent:

(1) $S$ is a chain of nil-extension of left strongly simple semigroups.

(2) $(\forall a, b \in S)\left(\exists n \in Z^{+}\right) a^{n} \in\left(S a^{m} b S a^{n}\right]$ or $b^{n} \in\left(S a b^{m} S b^{n}\right]$ for every $m \in Z^{+}$.

(3) $(\forall a, b \in S)\left(\exists n \in Z^{+}\right) a^{n} \in\left(S a b S a^{n}\right]$ or $b^{n} \in\left(S a b S b^{n}\right]$.

(4) $S$ is left $\pi$-semiregular and $\operatorname{LSReg}(S)$ is a chain of left strongly simple po-semigroups.

Proof. (1) $\Rightarrow(2)$ Let $\sigma$ be a semilattice congruence of $S$ such that $(x)_{\sigma}$ is a nil-extension of left strongly simple semigroup $K_{x}$ of $S$ for every $x \in S$ and $(S / \sigma, \preceq)$ is a chain. Let $a, b \in S$. For $(a)_{\sigma},(b)_{\sigma}$, we have $(a)_{\sigma} \preceq(b)_{\sigma}$ or 
$(b)_{\sigma} \preceq(a)_{\sigma}$. If $(a)_{\sigma} \preceq(b)_{\sigma}$, then $a, a b \in(a)_{\sigma}$, so for every $m \in Z^{+}$, we have $a, a^{m} b \in(a)_{\sigma}$. By Theorem 3.1, then $a^{n} \in\left((a)_{\sigma} a^{m} b(a)_{\sigma} a^{n}\right] \subseteq\left(S a^{m} b S a^{n}\right]$. If $(b)_{\sigma} \preceq(a)_{\sigma}$, in a similar way, we obtain $b^{n} \in\left(S a b^{m} S b^{n}\right]$ for every $m \in Z^{+}$.

$(2) \Rightarrow(3)$ It is obvious.

$(3) \Rightarrow(4)$ It is clear that $S$ is an Archimedean po-semigroup and $a^{n} \in$ $\left(S a^{2} S a^{n}\right]$ for every $a \in S$. Then there exist $x, y \in S$ such that $a^{n} \leq x a^{2} y a^{n} \leq$ $\left(x a^{2} y\right)^{k} a^{n}$ for every $k \in Z^{+}$. For $x a^{2} y, a^{n}$ there exist $u, v \in S$ and $m \in Z^{+}$ such that $\left(x a^{2} y\right)^{m} \leq u a^{n} v$. Now, we have $a^{n} \leq\left(x a^{2} y\right)^{m} a^{n} \leq u a^{n} v a^{n}$, which shows that $a^{n} \in \operatorname{LSReg}(S)$. Hence $S$ is left $\pi$-semiregular.

Let $a, b \in \operatorname{LSReg}(S)$. Then there exist $u, v \in S$ such that $a \leq u a v a \leq$ $(u a v)^{m} a$ for every $m \in Z^{+}$. Since $S$ is Archimedean, for $u a v, a b$ there exist $x, y \in S$ and $k \in Z^{+}$such that $(u a v)^{k} \leq x a b y$. Now we have $a b \leq(u a v)^{k} a b \leq$ $x a b y a b$, so $a b \in \operatorname{LSReg}(S)$, i.e., $\operatorname{LSReg}(S)$ is a subsemigroup.

On the other hand, $\operatorname{LSReg}(S)$ is simple. In fact: Let $a \in \operatorname{LSReg}(S)$, then there exist $u, v \in S$ such that $a \leq u a v a \leq u^{n} a(v a)^{n}$ for every $n \in$ $Z^{+}$. Since $S$ is left $\pi$-semiregular, for $u, v a$ there exists $k \in Z^{+}$such that $u^{k},(v a)^{k} \in \operatorname{LSReg}(S)$. Hence $a \in(\operatorname{LSReg}(S) a \operatorname{LSReg}(S)] \subseteq \operatorname{LSReg}(S)$, so $(L S R e g(S) a L S R e g(S)]=L S R e g(S)$.

Let $K=L S R e g(S), a, b \in K$. For $a, a b \in K$, then $K=(K a b K]$. Thus there exist $x, y, u, v \in K$ such that $a \leq x a y a \leq x u a b v y a \in(K a b K a]$. For $b, a b \in K$, in a similar way, we have $b \in(\bar{K} a b K b]$. By Theorem $3.1, K$ is a chain of left strongly simple po-semigroups.

$(4) \Rightarrow(1)$ Let $a, b \in S$. Since $S$ is left $\pi$-semiregular, then there exist $n, m \in Z^{+}$such that $(a b)^{n},(b a)^{m} \in \operatorname{LSReg}(S)$. For $(b a)^{m},(a b)^{n}$, by Theorem 3.1 , we have

$$
(b a)^{m} \in\left(\operatorname{LSReg}(S)(b a)^{m}(a b)^{n} \operatorname{LSReg}(S)(b a)^{m}\right] \subseteq\left(S a^{2} S\right]
$$

or

$$
(a b)^{n} \in\left(\operatorname{LSReg}(S)(b a)^{m}(a b)^{n} \operatorname{LSReg}(S)(a b)^{n}\right] \subseteq\left(S a^{2} S\right] .
$$

Thus $(a b)^{m n} \in\left(S(a b)^{m n} S(a b)^{m n}\right] \subseteq\left(S a^{2} S(a b)^{m n}\right] \subseteq\left(S a^{2} S\right]$. From this it follows by Lemma 1.2 that $S$ has Putcha's property. In view of Lemma $1.2, \mathcal{N}$ is the greatest semilattice congruence on $S$ such that $(x)_{\mathcal{N}}$ is an Archimedean subsemigroup for every $x \in S$. Since $\mathcal{N}$ is the least complete semilattice congruence on $S$, and $S$ is left $\pi$-semiregular, by Lemma $2.1,(x)_{\mathcal{N}}$ is left $\pi$-semiregular. From this it follows by Theorem 2.3 that $(x)_{\mathcal{N}}$ is a nil-extensions of left strongly simple $p o$-semigroups.

Moreover, Let $(a)_{\sigma},(b)_{\sigma} \in S / \mathcal{N}$. Since $S$ is left $\pi$-semiregular, then there exist $n, m \in Z^{+}$such that $a^{n}, b^{m} \in \operatorname{LSReg}(S)$. For $a^{n}, b^{m}$, by Theorem 3.1, we have

$$
a^{n} \in\left(L S R e g(S) a^{n} b^{m} \operatorname{LSReg}(S) a^{n}\right] \subseteq(S a b S] \subseteq I(a b)
$$

or

$$
b^{m} \in\left(L S R e g(S) a^{n} b^{m} L S \operatorname{Reg}(S) b^{m}\right] \subseteq(S a b S] \subseteq I(a b) .
$$


If $a^{n} \in(S a b S]$, then there exist $u, v \in S$ such that $a^{n} \leq u a b v$. Then $N(a) \ni$ $a^{n} \leq u a b v$, so we have $u a b v \in N(a)$, it is implies $a b \in N(a)$, we get $N(a b) \subseteq$ $N(a)$. If $b^{m} \in(S a b S]$, in a similar way, we have $N(a b) \subseteq N(b)$. On the other hand, $a b \in N(a b)$, we have $a, b \in N(a b)$. Therefore, $N(a) \subseteq N(a b)$ and $N(b) \subseteq N(a b)$. Thus, we have $N(a b)=N(a)$ or $N(a b)=N(b)$ i.e., $(a)_{\mathcal{N}}=(a b)_{\mathcal{N}}$ or $(b)_{\mathcal{N}}=(a b)_{\mathcal{N}}$. We have $(a)_{\mathcal{N}} \preceq(b)_{\mathcal{N}}$ or $(b)_{\mathcal{N}} \preceq(a)_{\mathcal{N}}$.

\section{References}

[1] G. Birkhoff, Lattice Theory, Vol. XXV, American Math. Soc. Coll. Publ., Providence, 1967.

[2] Y. Cao, On weak commutativity of po-semigroups and their semilattice decompositions, Semigroup Forum 58 (1999), no. 3, 386-394.

[3] Y. Cao and X. Xu, Nil-extensions of simple po-semigroups, Comm. Algebra 28 (2000), no. 5, 2477-2496.

[4] A. H. Clifford, Extensions of semigroups, Trans. Amer. Math. Soc. 68 (1950), 165-173.

[5] A. H. Clifford and G. B. Preston, The Algebraic Theory of Semigroups. Vol. I, Mathematical Surveys, no. 7, American Mathematical Society, Rhode Island, 1964.

[6] N. Kehayopulu, Note on Green's relations in ordered semigroups, Math. Japon. 36 (1991), no. 2, 211-214.

[7] _ Remark on ordered semigroups, Math. Japon. 35 (1990), no. 6, 1061-1063.

[8] _ On intra-regular ordered semigroups, Semigroup Forum 46 (1993), no. 1, 271278.

[9] _ On completely regular poe-semigroups, Math. Japon. 37 (1992), no. 1, 123-130.

[10] N. Kehayopulu and M. Tsingelis, Ideal extensions of ordered semigroups, Comm. Algebra 31 (2003), no. 10, 4939-4969.

[11] _ Remark on ordered semigroups, Sovremennaja Algebra, St. Petersburg Gos. Ped. Herzen Inst., 1992, 56-63.

[12] S. K. Lee and Y. I. Kwon, On left, right weakly prime ideals on po-semigroups, Commun. Korean Math. Soc. 11 (1996), no. 2, 315-321.

[13] M. Petrich, Introduction to Semigroups, Merrill Research and Lecture Series, Charles E. Merrill Publishing, Ohio, 1973.

[14] Q. S. Zhu, A note on left (right) semiregular po-semigroups, Far East J. Math. Sci. 26 (2007), no. 3, 697-704.

[15] _ On characterizations of completely regular ordered semigroups, Southeast Asian Bull. Math. 29 (2005), no. 4, 827-834.

[16] Q. S. Zhu and T. F. Qing, On kernel in ordered semigroups, Journal of Information Engineering University(China) 9 (2008), no. 4, 492-494.

Department of Applied Mathematics and Physics

InSTITUTE OF SCIENCES

INFORMATION ENGINEERING UNIVERSITY

ZhengZhou 450001, P. R. ChinA

E-mail address: qingshunzhu@yahoo.com.cn 